\title{
MELIPONARIO SIPASS - UNA EXPERIENCIA CON LA ABEJA ANGELITA TETRAGONISCA ANGUSTULA CON DOS TIPOS DE COLMENAS RACIONALES EN EL DE CEAD ACACIAS
}

Meliponario SIPASS - an experience with the angelita beetle Tetragonisca angustula with two types of rational hives in CEAD Acacias

\section{Dayro Enrique Cortes Martínez}

Esp. en Educación Superior a Distancia Universidad Nacional Abierta y a Distancia - ECAPMA dayro.cortes@unad.edu.co

\section{Oscar Javier Olarte Blandon}

Magister en Administración de Organizaciones

Universidad Nacional Abierta y a Distancia - ECAPMA oscar.olarte@unad.edu.co

\section{RESUMEN.}

En el año 2012 se inició con trabajos de investigación con la abeja melipona Tetragonisca angustula conocida como abeja angelita; una de las más presentes en la región tanto en el urbanismo (viviendas) como en la ruralidad (bosques).

El interés se suscita a partir de que es una de las más asediadas por las personas para obtener su miel, provocando eliminación total de las colonias por el desconocimiento de su dinámica biológica. Son estas las razones que motivaron a investigar de qué manera se podían manejar racionalmente; es así que se llega a la práctica de la meliponicultura una actividad ancestral y desconocida en los actuales esquemas de producción.

La primera actividad que se genera es la construcción de las instalaciones del meliponario, construcción de cajas racionales que permitieran las buenas prácticas de manejo y reproducción y el inpacto de este trabajo con la comunidad

Este documento presenta una secuencia de trabajo que ha permitido avanzar en la apropiación y el compartir de saberes en los años

DOI: https://doi.org/10.22490/ECAPMA.3511 
subsiguientes, relacionado con el uso de dos tipos de colmenas verticales la colmena INPA y la colmena inteligente AF y comunidades.

Palabras Clave: Abejas; Cajas racionales; Meliponicultura; Tatragonisca angustula.

\section{ABSTRACT.}

In 2012, he began with research work with the Tetragonisca angustula melipona bee known as the angel bee; one of the most present in the region in both urban planning (housing) and rurality (forest).

The interest is aroused since it is one of the most besieged by people to obtain their honey, causing total elimination of the colonies due to ignorance of its biological dynamics. These are the reasons that motivated to investigate how they could be handled rationally; Thus, an ancestral and unknown activity in the current production schemes is reached in the practice of meliponiculture.

The first activity that is generated is the construction of the facilities of the meliponario, construction of rational boxes that allowed good management and reproduction practices and the impact of this work with the community

This document presents a sequence of work that has allowed progress in the appropriation and sharing of knowledge in subsequent years, related to the use of two types of vertical hives, the INPA hive and the intelligent Hive AF and communities.

Keywords: Bees; Rational boxes; Meliponiculture; Tatragonisca angustula.

\section{INTRODUCCIÓN.}

La meliponicultura es la cría técnica de la abeja melipona en sus diferentes especies en condiciones controladas que permiten su explotación de manera racional, eficiente y con equilibrio en los ecosistemas. Esta práctica es ancestral ya que estas especies son propias de gran parte del continente americano y franja tropical En la actualidad el ambiente natural de estas abejas está amenazado por la indiscriminada deforestación, el 
avance de la frontera agrícola y uso de agrotóxicos, y desconocimiento de su biología. Los sitios de nidificación de los meliponinos son cada vez más reducidos; es importante resaltar que las abejas juegan un papel fundamental en la polinización que asegura la formación de frutos y semillas y en consecuencia la disponibilidad de recursos alimenticios provenientes del reino vegetal.

Este documento relata y contextualiza experiencias del meliponario SIPASS de la UNAD CEAD de Acacias en referencia a el uso de dos tipos de colmenas para la abeja Angelita Tetragonisca angustula y su impacto.

\section{METODOLOGÍA.}

\section{Investigación Bibliográfica}

La investigación se fundamentó mediante el uso de base de datos web of cience, scopus y Google socollar y gestores de referencia como el Zotero y Mendeley que permitieron acceder a documentos que ampliaron y fundamentaron los avances teóricos sobre el tema.

\section{Investigación de campo}

Esta se viene realizando en el Meliponario del Cead Acacias liderado por el semillero de investigaciones y Agrostología SIPASS, que inició sus con el uso de cajas racionales modelo INPA y luego de cinco años de seguimiento se continuó con la evaluación y validación de la caja racional AF. Se han desarrollado divisiones, colecta de productos, ejercicios de trampeos para las Meliponas menores abeja angelita Tetragonisca angustula y socializaciones con las comunidades interesadas en el tema.

\section{Aspectos básicos de la meliponicultura.}

\section{Meliponicultura}

González et al 2004 describen la meliponicultura como el cultivo de las abejas nativas sin aguijón actividad que se remonta a tiempos anteriores a la llegada de los españoles a América.

La Meliponicultura se refiere a la cría y manejo de abejas sin aguijón y recibe este nombre debido a que a este tipo de abejas se clasifica taxonómicamente dentro de la tribu Meliponini, que corresponde a uno de los muchos grupos de abejas nativas de América. Harriet 1999.

La meliponicultura concepto que refiere el aprovechamiento racional y tecnificado de las abejas sociales de la familia apidae, subfamialia apinae, 
tribu meliponini y de estas se desprenden dos grupos las Meliponas que corresponden a abejas mas grandes que tienen tamaños de 7 a $15 \mathrm{~mm}$ y las trigonas que son abejas pequeñas, delgadas de tamaños de 2 a 11 $\mathrm{mm}$. Las diferencias de estos dos grupos radican en especial a la forma en cómo se produce o se forma la reina. Villas-Bôas, J. (2012). Este grupo de abejas carecen de aguijón y sus nidos se estructuran de manera horizontal.

Son el único grupo de abejas nativo de América que posee comportamiento altamente social, colonias numerosas y perennes que se reproducen por medio de enjambres y que cuentan con diferenciación de castas (reina, obreras y zánganos) y una comunicación altamente desarrollada entre los miembros de la colonia. Harriet 1999.

Existe una confusión del significado de colmena y colonia; dos términos que parecen sinónimos pero que en realidad difieren. La colonia es el material biológico que corresponde a la población que esta constituida por castas de abejas, conocidas como obreras, zánganos y reina. Vic, P 2004. Por otro lado, colmena es el habitáculo o la infraestructura artificial, diseñada de acuerdo a las necesidades de las abejas para que estas se puedan aprovechar racionalmente y se potencialice su inclusión en los sistemas de producción. Existen variados modelos como los MARIA y UTOB Domínguez \& Ruiz (2002); colmena INPA, Coletto \& Freire (2006). Colmena PNN Pablo Nogueira Neto Fonte et al (2013), la colmena inteligente AF en alusión a su diseñador el meliponicultor Ailton Fontana entre otras.

Es indudable y vale reconocer que estos insectos prestan un real servicio ecosistémico importante, entendiendo tal concepto como todos aquellos beneficios que la sociedad obtiene de los ecosistemas, Corredor et al. 2012.

Las abejas Meliponas como recurso Zoogenético participa en el escenario de la agrodiversidad para contribuir con la polinización; una de las dinámicas ecosistémicas más relevantes que garantizan la seguridad alimentaria. Bonilla et al. 2013 ponen de manifiesto que en la agrodiversidad interactúan los recursos fitogenéticos, zoogenéticos, microorganismos y hongos

\section{Decaimiento de la meliponicultura}


Medellin et al 1990 le atribuyen el decaimiento de la meliponicultura (cultivo de abejas sin aguijón) al arribo de la abeja europea Apis mellifera cuya producción masiva de miel desplazó en gran parte el sistema tradicional que lo constituían las abejas meliponas. También de manera paralela la introducción de la caña de azúcar y su consumo generalizado contribuyeron aún más al abandono de esta actividad.

La escasa tecnificación de la meliponicultura ha hecho que esta sea poco conocida y tenida en cuenta como sistema de producción. Así que quienes cosechaban la miel terminaban eliminando sus nidos $\mathrm{y} / \mathrm{o}$ colonias $\mathrm{y}$ reduciendo la población natural que amenaza su presencia y equilibrio natural.

La incorporación de cajas racionales (colmenas) permite el manejo en la producción y reproducción y permite su fácil revisión

\section{¿Que debo conocer para trabajar con Meliponas?}

Es importante resaltar que para trabajar con Meliponas al igual que con otras especies de interés zootécnico se debe conocer por menores relacionados con su biología, comportamiento, requerimientos y de esta manera darle manejo técnico y aprovechar todo su potencial. Estos aspectos son de suma importancia y por tal motivo se hará un viaje somero para que se tengan elementos base para emprender la hermosa actividad de la meliponicultura.

En este caso específico se presentará una descripción de dos elementos importantes en esta actividad; por un lado, lo referente a la colonia entendida como el material biológico constituido por los individuos (reina, obreras y zángano) y el nido con cada una de sus partes (panales, involocro, cerumen, etc.) y la colmena entendido como la infraestructura donde se aloja la colonia (cajas racionales).

\section{Conociendo Partes del Nido}

El nido de las abejas Meliponas entre ellas la abeja angelita (tetragonisca angustula) presenta características similares en todos sus componentes y para efectos didácticos lo presentaremos en dos partes; la primera relacionada con los individuos (reina, obrera y zángano) y la segunda con la infraestructura de su nido.

\section{Individuos de la colonia.}


Está conformado por la reina que es la hembra fértil que se dedica a la reproducción; es la única que pone huevos para mantener la población y garantiza la supervivencia de las próximas generaciones; las obreras son las abejas que realizan los trabajos de recolección de néctar, polen, agua, resinas vegetales, semillas, arcilla que usa para la construcción del nido, alimentación propia y la de la demás población y los y por último los zánganos: individuos dedicados a fecundar a las nuevas reinas Tuso 2014. El ciclo de desarrollo de esta especie es de 36 días, donde según (Nates, 2006), el huevo dura 6 días, la larva y pupa tiene una duración de 10 y 20 días respectivamente.

\section{Infraestructura del nido}

Las abejas sin aguijón construyen sus nidos y establecen sus colonias en arboles huecos, paredes y cualquier lugar que le provea de un espacio libre de humedades excesivas y presencia de hormigas y otras especies que le representen peligro, estos espacios lo adecúan para su habitad. Las principales partes del nido son: Piquera o entrada cuyas medidas oscilan de $0.7-0.9 \mathrm{~cm}$ de diámetro y una profundidad de $1-3.5 \mathrm{~cm}$. Alarcón \& Ibáñez (2008) figura 1 , , potes de almacenamiento que están construidos de cerumen y donde almacenan la miel y el polen que son sus fuentes principales de energía y proteína, , batumen que es un material rígido que se encuentra generalmente cuando los espacios anidados tiene alto riego de humedad y por esta razón mezclan barro, resinas vegetales y semillas repelentes para neutralizar estos riesgos y también lo usan también para sellar aberturas., el involucro está formado por delgadas láminas de cerumen, que las abejas operarias elaboran y disponen en varias capas en torno al área de cría, con el fin de conservar la temperatura adecuada para huevos, larvas y pupas que están prontas a nacer en la colmena. Estas láminas tienen pequeñas separaciones, que se convierten en las vías de acceso de las abejas operarias y la Reina al área de cría. Alarcón \& Ibáñez (2008) figura 2. los discos de cría (figura 3) son los panales construidos de cerumen compuestos por varios alveolos dispuestos de modo horizontal, a manera de pisos de un edificio. Los alveolos son elipsoidales y dispuestos unos al lado de otro en forma ordenada, separados soportados por columnas hechas de cerumen. Los alveolos son utilizados para la ubicación de los huevos, larvas y pupas, de donde emergen las abejas jóvenes; inicialmente las operarias colocan el alimento suficiente para el desarrollo de las larvas luego la reina deposita el huevo en cada alveolo con alimento y posteriormente otras operarias 
se encargan de sellarlos hasta que las abejas emerjan o nazcan. Los discos de cría que están compuestos por alveolos con una tonalidad más oscura son aquellos donde recientemente han sido sellados (cría inmadura) demorándose más tiempo en emerger las abejas y los discos que tienen una tonalidad más clara indican que las abejas están próximas a emerger (Cría madura). También construyen alveolos más grandes dispuestos en los bordes de los panales donde son colocados los huevos de las princesas o futuras reinas. Alarcón \& Ibáñez (2008), adaptado por Cortes \& Olarte 2019; el basurero es un área pequeña en la parte inferior del nido donde las abejas expulsan sus desechos. Está ubicado fuera de las zonas de cría y de almacenamiento de alimento. Alarcón \& Ibáñez (2008).

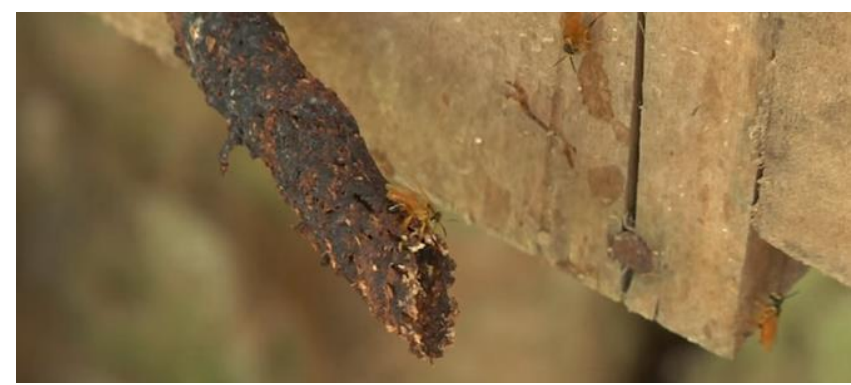

Figura 1. Piquera

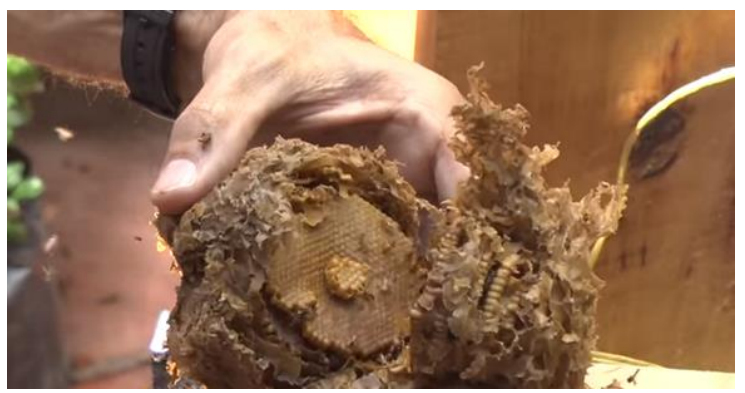

Figura 2. Involucro del nido 


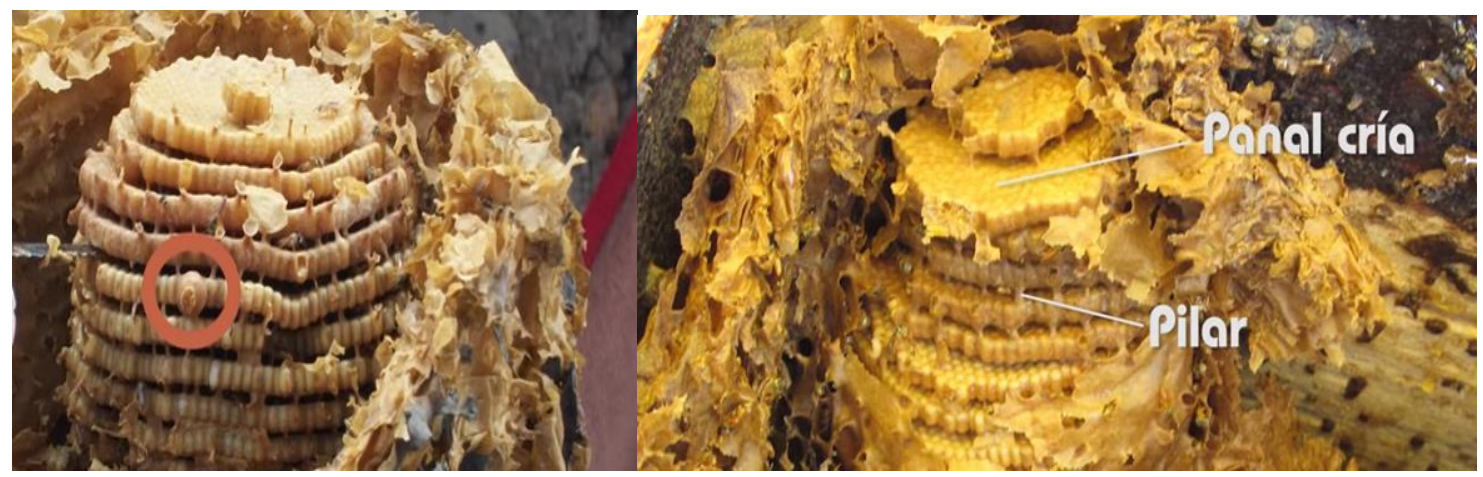

Figura 3. Nido - panal de cría - pilares

\section{Colmenas (cajas racionales) para abejas Meliponas.}

La incorporación de las cajas racionales (colmenas) facilita los trabajos en la meliponicultura tanto el manejo del material biológico (colonias) en lo referente a divisiones y monitoreos y manejo de la producción (cosecha eficiente) de la miel y otros productos como el polen, propóleos y cerumen.

Actualmente existen varios modelos de cajas racionales, las más empleadas son aquellas cajas con divisiones o alzas y construidas con madera de buen grosor $(2.5 \mathrm{~cm}$ preferiblemente) y bien seca. Lo importante de estas cajas es que ofrezcan a las abejas un espacio o sitio ideal, donde no sean afectadas sus condiciones zootécnicas debido a bajas temperaturas en las noches o durante el invierno y lo más importante es que sea de difícil acceso para las plagas y/o enemigos (Baquero \& Stamatti, 2007).

Se han propuesto dos modelos relevantes las cajas de tipo vertical y horizontal; pero basados en nuestra experiencia haremos referencia a las cajas o colmenas verticales, criterio adoptado por que este sigue el patrón natural de los panales de cría en troncos de árboles.

Lo que concierne a este documento se trabajaron con las cajas racionales o colmenas INPA y Colmena inteligente $A F$, la primera fue propuesta por el investigador brasilero Virgilio Angola Portugal Araujo en 1955. Rovira; Tschirsch \& Schvezov 2005.

\section{Colmena INPA}


Esta colmena cuenta con tres módulos; los dos primeros están dispuestos para alojar a la zona de cría (colonia), y el nido. El módulo superior (alza - melgueira) para que las abejas alojen los potes de miel, que desde luego facilita la colecta de miel de una forma eficiente, higiénica y sin mayor perturbación a la población (colonia) ya que incluso se puede separar de la colmena por su disposición divisoria (secciones) para minimizar riesgos y facilitar el trasporte. Esta característica modular permite una eficiente evolución de las colonias para su fortalecimiento y posteriores divisiones o enjambrazones que favorecen su conservación y servicio a la producción pecuaria en producción de miel y servicios ecosistémicos.

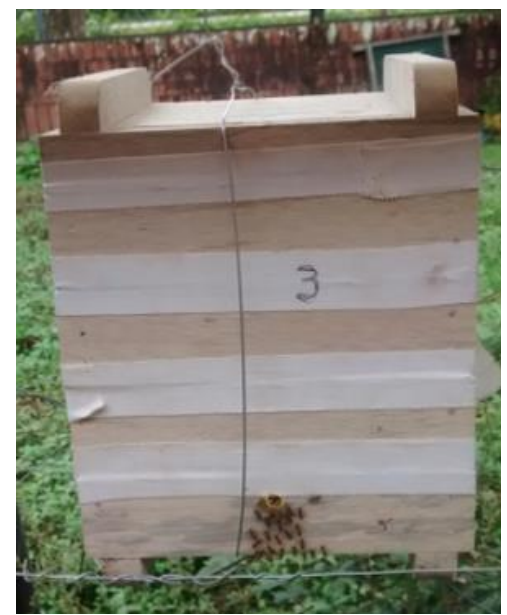

Figura 4. Caja racional INPA meliponario SIPASS

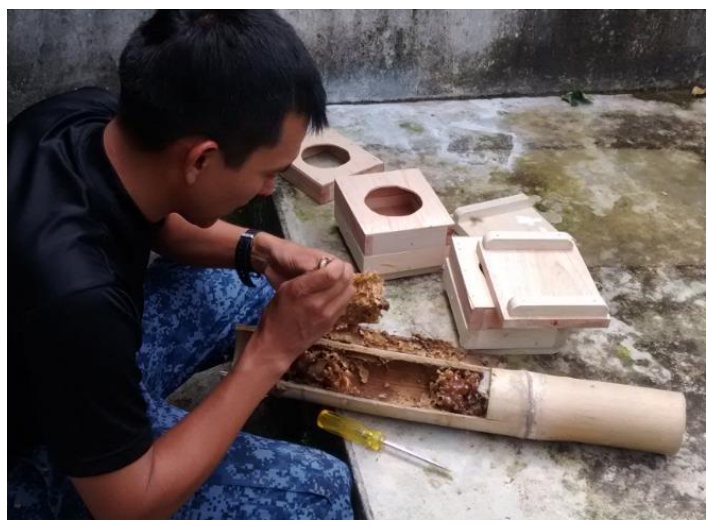

Figura 5. Trasiego de nido de tetragonisca angustula a caja racional INPA. 


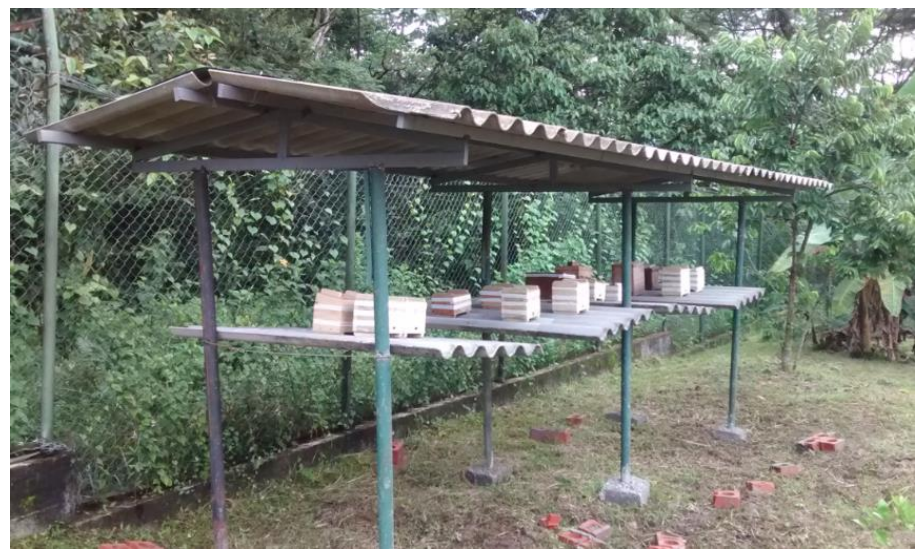

Figura 6. Meliponario SIPASS con colmenas tipo INPA

\section{La colmena o caja racional AF}

Esta colmena es muy práctica que fue desarrollada por el Meliponicultor brasilero Ailton Fontana de la ciudad de Novo Horizonte, SP. Quien la maneja con éxito en su meliponario Flamboyant y consta de las siguientes partes:

Presenta 5 gavetas dos destinadas para la cría; un denominado fundo y la segunda como sobrenido.

Las gavetas 3,4 y 5 son destinadas como alzas de miel.

Cada gaveta esta está confeccionada en madera seca de 1,5 a 2 centímetros de espesor por 5 centímetros de altura. Todas ellas de una sola medida en el fondo de cada gaveta presentan un cuadriculado de alambre o nylon de pesca, fijado con puntillas de cabeza o ganchos de papelera, para servir como especie de piso, tanto para los discos de cría y para los potes de miel y de polen. 


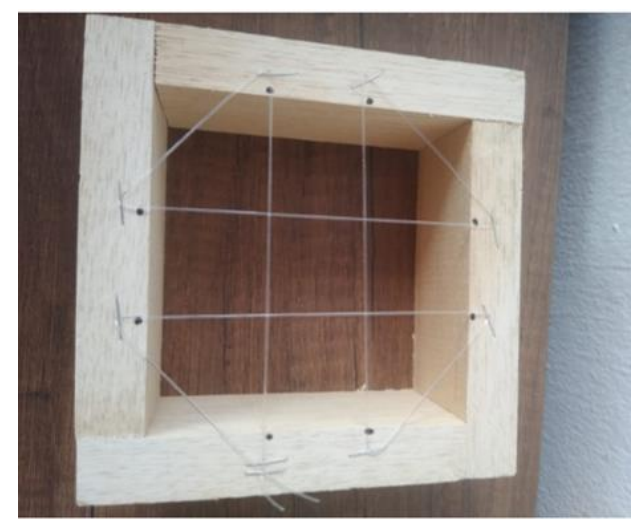

Figura 7. Piso en alambre divisorio para gavetas.

Entre gaveta y gaveta se disponen dos láminas de acetato en el caso de las gavetas uno y dos (fundo y sobrenido) estas tienen contienen un solo agujero de 60 milímetros al centro, destinado al paso de los discos de cría. Ver figura 8 y 9.

En las gavetas 3,4 y 5 los acetatos tienen otra disposición que es la presencia de huecos triangulares en la esquina que permiten el paso de las abejas hacia la parte superior para que almacenen la miel y el polen. Ver figura 13. El ultimo acetato que va en la parte superior de la última gaveta o alza de miel (numero 5) no posee ningún agujero esto permite cerrar el conjunto de gavetas. Ver figura 9.
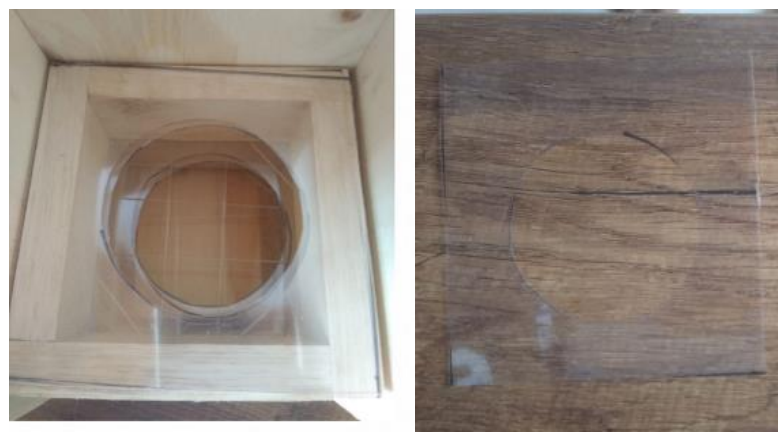

Figura 8. Acetato divisorio gavetas fundo y sobrenido. 


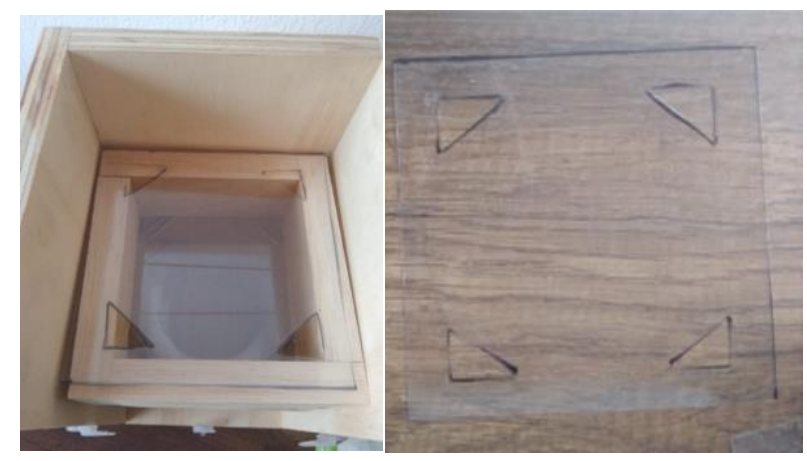

Figura 9. Acetato divisorio gavetas de alzas de miel gavetas 3,4 y 5 .

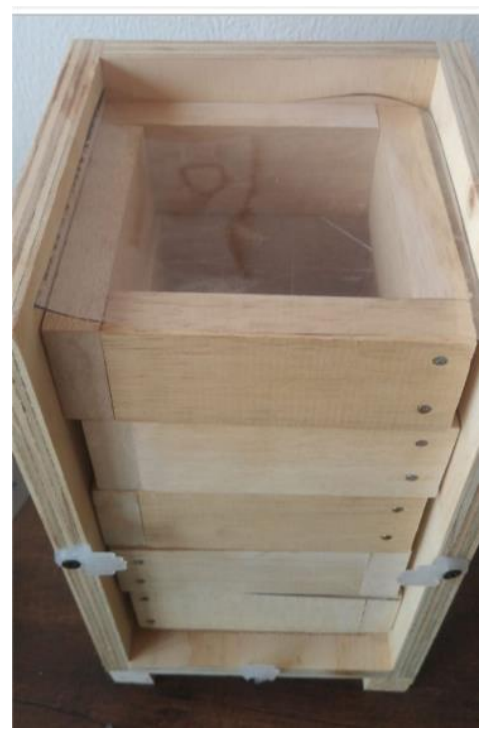

Figura 10. Acetato divisorio de la última gaveta sin agujeros.

\section{Cubierta:}

La cubierta es La estructura que protege las gavetas de medio externo tiene una altura de $30 \mathrm{~cm}$ de alto por $17 \mathrm{~cm}$ de anclo cuenta con tapa posterior, subtapa y tapa superior que hermetizan las gavetas y protegen a las abejas de interferencias externas. 


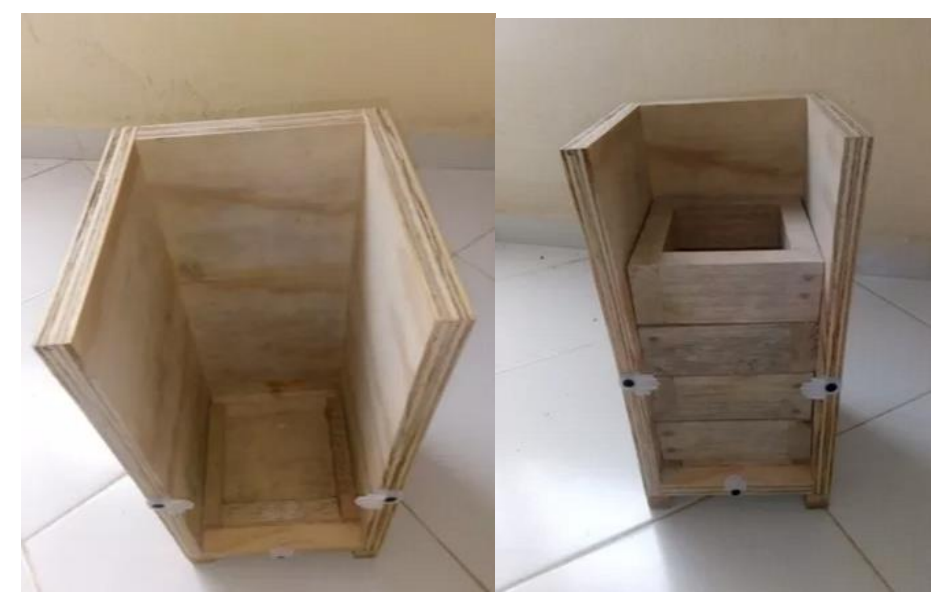

Figura 11. Cubierta externa Colmena inteligente AF

Se puso a disposición un video explicativo de esta colmena en YouTube que puedes observar haciendo clic https://www.youtube.com/watch? $v=-$ PLqTtbIUIY\&t $=195 \mathrm{~s}$.

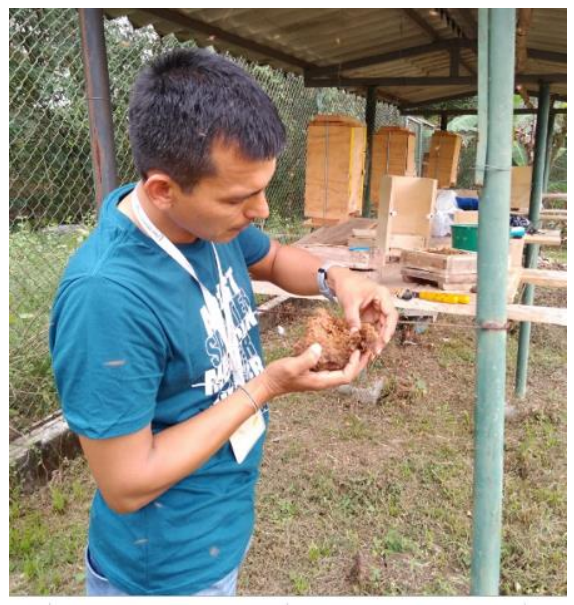

Figura 12. Trabajo de campo Meliponario SIPASS Colmenas AF

\section{RESULTADOS Y DISCUSIÓN}

\section{Experiencias con colmenas INPA}

El proyecto inicio con 4 colmenas tipo INPA y de estas se obtuvieron 6 divisiones aumentado el número de colmenas con sus respectivas colonias a 10. La recolección de miel se realizó en dos temporadas: 1 temporada 
entre los meses de diciembre y febrero y la segunda temporada entre los meses de julio y agosto; esto coincide con las bajas precipitaciones y aumento de la floración del área de estudio. La producción de miel por cosecha fue en promedio de 250 a 400 centímetros cúbicos en la primera temporada y de 200 a $320 \mathrm{~cm}$ para la segunda temporada.

De esta primera experiencia con este tipo de colmenas se presentaron dificultades en relación a:

Ingreso de hormigas por los espacios entre modulo y modulo, esto conlleva al uso de cintas de enmascarar (papel) lo que implica costos adicionales.

Se usó como separador entre modulo y modulo láminas de triplex de madera que las abejas propoleolizaban y al momento de hacer manejos se prolongaban los tiempos de cada actividad, sufriendo daños de la estructura de cada compartimiento.

\section{Experiencias con colmenas inteligentes AF}

Este modelo de cajas permitió un avance significativo en el meliponario ya que se progresó en aspectos técnicos como:

Control más efectivo de enemigos externos de las abejas (hormigas, foridos entre otros)

Facilidad de manejo en las revisiones periódicas.

Efectividad en las divisiones de las colonias

Efectividad en la cosecha de la miel que fueron muy similares a las obtenidas con la colmena INPA, con la colmena AF en la primera temporada la producción de miel por cosecha fue en promedio de 250 a 420 centímetros cúbicos y de 200 a $370 \mathrm{~cm}$ para la segunda temporada.

Otro resultado importante en este seguimiento del meliponario SIPASS fue la incorporación de la captura de enjambres por métodos de trampeos que consiste en el uso de receptáculos artificiales embadurnados con atrayentes que permiten que colonias en proceso de enjambrazón sean atraídas y aniden en ellos. Esto favorece en gran manara la reproducción natural de las abejas Meliponas y reduce los impactos que para obtener estas colonias realizan las personas como tumba de árboles, rompimiento de paredes que en muchos de los casos terminan con la eliminación de los nidos y su población. 
Esta metodología consiste en el uso de botellas Pets (gaseosa de 1.5) embadurnadas con una mezcla liquida que se genera a partir de la mezcla de geopropóleo y alcohol en el interior de la botella; esta a su vez se envuelve con papel periódico y una bolsa negra para protegerla y generar un ambiente adecuado para las abejas como lo es una temperatura adecuada y oscuridad. En la tapa de la botella se abre un orificio que servirá de piquera para el ingreso de las abejas atraídas. Para compartir este método el semillero puso al servicio un video de consulta en Yo tobe que puedes observar en el siguiente enlace: https://www. youtube.com/watch? $v=X L C F S w N o S H U \& t=33 \mathrm{~s}$, que hasta la fecha tiene mas de 1300 visitas.

Estas experiencias nos han permitido avanzar en trasferir estas experiencias a estudiantes, comunidades y colegios para que emprendan esta loable labor es así que en el año 2018 se organizó con la secretaria de fomento del municipio de Acacias Meta el curso Taller Meliponicultura - promoviendo el fomento la conservación y la inclusión de la abeja Tetragonisca angustula o Angelita a los sistemas de producción del municipio" donde asistieron 22 participantes se promovió esta actividad y se invitó a una explotación más racional basado en la técnica.

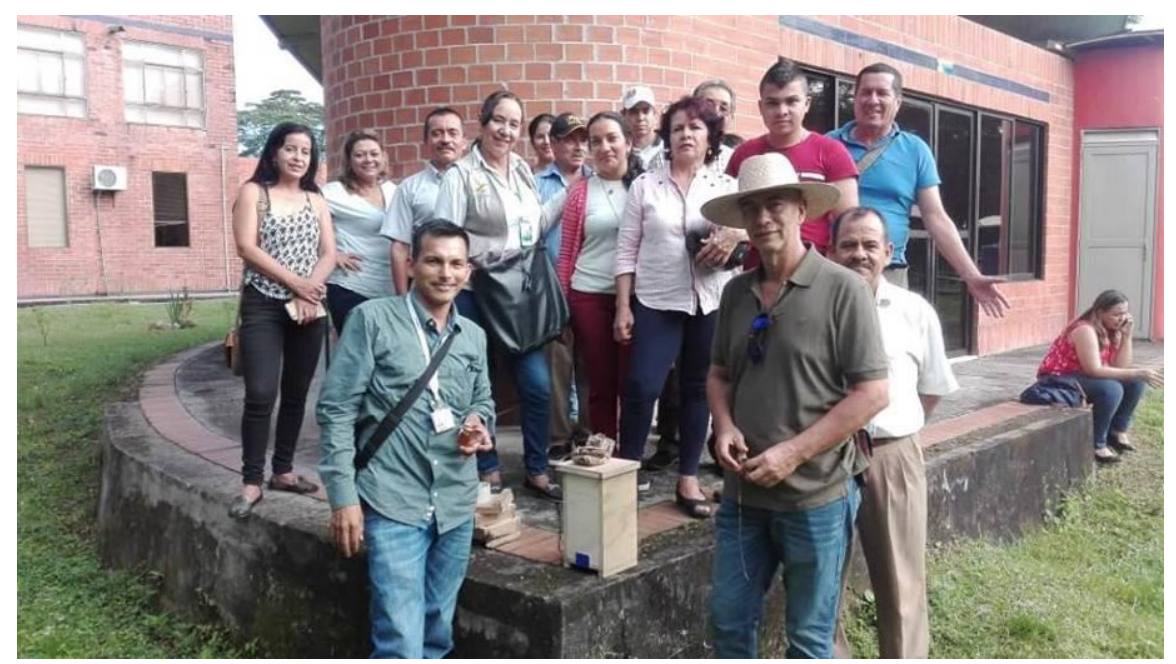

Figura 13. Curso Meliponicultura Acacias 2018.

Globalizar los conceptos es transferirlos directamente in situ o por medio de las TICs como lo manifiesta Martínez Giron 2018 donde cita a Cartea 
\& Meira, 2006. "La globalización, se presenta como una forma de difusión más leve o ligera en la que aparecen nuevas tecnologías para la comunicación, nuevas formas de reproducción del capital y nuevas estrategias de producción".

Es indiscutible el papel que juegan las abejas en la producción de alimentos ya que estas son las encargadas de polinizar y de ello depende la producción de semillas y frutos. El aumento significativo de frutos se debe a la capacidad polinizadora ejercida por las abejas en los diferentes cultivos, en estudios como los referidos por Dusan Huaca 2017, hacen referencia a que el tamaño y el peso del grano de café son variables que se pueden ver afectadas por las características agroecológicas y de manejo aplicadas al cultivo. Sin embargo, es importante reflexionar que para alcanzar estos umbrales no solo basta mejoramiento genético y selección de progenies; sino también de la presencia de polinizadores.

\section{CONCLUSIONES.}

Es importante resaltar que es necesario fomentar la actividad de la meliponicultura como una opción productiva en los sistemas de producción, actividad que se había desvanecido por la incorporación de la apicultura en la década de los 50 con la abeja Apis mellifera. Esto conllevo a la casi desaparición cultural; tanto que las nuevas generaciones la desconocían en el sentido estrictamente teórico porque en realidad esta actividad es propia de nuestra historia; antes de la llegada de los europeos en la conquista los nativos ya la cultivaban y usaban la miel como alimento y medicina.

Estos escenarios de investigación - hacer y trasferir nutren ese propósito de hacer extensivo la invitación a recuperar y a la vez tecnificar la meliponicultura para avanzar en lo económico, agroecológico y restaurar la biodiversidad de nuestro bosques, repoblándolos de este preciado y necesario insecto "las abejas".

EI Meliponario SIPASS cuenta con la experiencia necesaria para continuar realizando capacitación en temas de interés general y promover la actividad como el eslabón perdido de producción de productos de la abeja; una abeja autóctona, inofensiva.

La meliponicultura del ahora debe basarse en el conocimiento detallado de cada elemento de su forma de ejercerla de manera eficiente y 
sostenible. Se debe promover su incorporación a los sistemas de producción de manera masiva comprendiendo aspectos de su biológica, manejo, infraestructura, reproducción sostenible y cosecha de sus productos inocuos para el servicio del ser humano en toda su expresión.

Creemos que el comienzo de ello es a través de la investigación y que estas experiencias sean compartidas para contribuir y mejorar sus múltiples formas de explotación racional, tratando de conservar y aumentar las poblaciones mediante técnicas integrales.

Se ha validado la efectividad del uso de cajas racionales o colmenas que ha facilitado la reproducción y fácil cosecha de los productos.

\section{BIBLIOGRAFÍA.}

Alarcón Sorto, R. C., \& Ibañez Salazar, L. C. (2008). Determinación de las características fisicoquímicas de la miel producida por las especies de abejas sin aguijon: Melipona beecheii (Jicota) y tetragonisca angustula (Chumelo) de Meliponicultores de la zona norte del Departamento de Chalatenango (Doctoral dissertation, Universidad de El Salvador).

Baquero, L., \& Stamatti, G. (Julio de 2007). Cría y manejo de abejas sin aguijón. Tucumán, Argentina: Subtrópico.

Bonilla Morales, M., \& Caetano, C. (2013). Inventario y valoración de la flora utilizada por la vereda Santa Teresa, Palmira (Valle del Cauca). Revista de Investigación Agraria y Ambiental, 4(1), 89-99. doi:https://doi.org/10.22490/21456453.985

Coletto-Silva, A., \& Freire, D. D. C. (2006). Hololepta (Leionota) reichii Marseul (Coleoptera, Histeridae) a new natural enemy for the meliponiculture in the Central Amazon, Brazil. Revista Brasileira de Zoologia, 23(2), 588-591.

Corredor Camargo, E., Fonseca Carreño, J., \& Páez Barón, E. (2012). Los servicios ecosistémicos de regulación: tendencias e impacto en el bienestar humano. Revista de Investigación Agraria y Ambiental, 3(1), 77-83. doi:https://doi.org/10.22490/21456453.936

Dominguez, L., Alfredo, D., \& Ruiz, B. (2002). Validación de dos modelos de colmenas MARIA y UTOB con abejas sin aguijón Melipona beecheii y Tetragonisca angustula, en El Paraíso, Honduras (No. T1551). ESCUELA AGRICOLA PANAMERICANA, . 
Dussan Huaca, I. (2017). Análisis de un modelo agroforestal cafetero en el municipio de Valparaíso, Caquetá, Colombia.. Revista de Investigación $\begin{array}{lllll}\text { Agraria } y & \text { Ambiental, } & \text { 8(1), } & 195 & -203 .\end{array}$ doi:https://doi.org/10.22490/21456453.1849

González, J. y otros. 2004. Rescate y desarrollo de meliponicultura tecnificada. I Reunión Estatal de Investigación Agropecuaria y Forestal. Mérida, Yucatán. Pag. 141-154.

Harriet J. de Jong. 1999. "The land of corn and honey: The keeping of stingless bees (meIiponiculture) in the ethno-ecological environment of Yucatan (Mexico) and EI Salvador". PhD-thesis. Utrecht University, pag. 423

Martínez Girón, J., Marin Rivera, J., Murillo Lopera, K., \& Rodríguez Delgado, I. (2018). La agroecología: alternativa de desarrollo sustentable ante la crisis ambiental en un mundo globalizado. Revista de Investigación Agraria y Ambiental, 9(2), 63 - 76. doi:https://doi.org/10.22490/21456453.2196

Medellín M. S., L. E. Campos, J. A. González, y G.V. Cámara. 1990. Meliponicultura Maya: Perspectivas para su Sostenibilidad, Sostenibilidad Maya-Universidad de California Riverside y FMVZUniversidad Autónoma de Yucatán. Mérida, Yuc

Nates, G. 2006. Abejas corbiculadas de Colombia. Himenóptera: Apidae, Universidad Nacional de Colombia, Sede Bogotá, Colombia.15-16p.

Rovira, E; Tschirsch, J; Schvezov, C. 2005. Características y cría de las yateí y otras meliponas. Material de divulgación. Este material ha sido elaborado con los aportes empíricos y técnicos de: Miembros de la Asociación de Productores de Yateí de Misiones (APYM), Fidel Godoy del CEDIT. Sergio Feversani del INTA Oberá. Provincia de Misiones, Argentina

Tuso, A., \& Jannet, D. 2014. Evaluación de los cambios pre y postcosecha de la miel de especies de abejas sin aguijón (Doctoral dissertation, Universidad Nacional de Colombia).

Villas-Bôas, J. (2012). Manual tecnológico: mel de abelhas sem ferrão.

Vit, Patricia. (2004). Productos de la colmena recolectados y procesados por las abejas: Miel, polen y propóleos. Revista del Instituto Nacional de 
Higiene Rafael Rangel, 35(2), 32-39. Recuperado en 13 de noviembre de 2019, de http://ve.scielo.org/scielo.php?script=sci_arttext\&pid=S079804772004000200006\&lng=es\&tlng=es. 